\title{
Vulnerabilidade social e a cárie dentária na primeira infância
}

\section{Social vulnerability and dental care in first childhood}

DOI: $10.46919 / \operatorname{archv2n6-006~}$

Recebimento dos originais: 01/06/2021

Aceitação para publicação: 31/07/2021

\section{Amanda Albuquerque Diniz}

Graduação em Odontologia - Universidade de Fortaleza - Unifor

E-mail: amandadiniz94@yahoo.com.br

\section{Isabelle Monteiro Da Silva Lima}

Graduação em Odontologia - Universidade de Fortaleza - Unifor

E-mail: isabellemontteiro@gmail.com

\section{Karyne Barreto Gonçalves Marques}

Especialização em Saúde Coletiva e Odontopediatria - Universidade Estadual do Ceará

E-mail: karynebgmarques@ hotmail.com

Luiz Carlos Costa Madeira Alves

Mestrado em Odontologia - Universidade de Fortaleza - Unifor

E-mail: uizcarloscostamalves@gmail.com

Ingrid Cordeiro Monte

Mestrado em Odontologia - Universidade de Fortaleza - Unifor

E-mail: ingrid_monte_@hotmail.com

\section{Lucianna Leite Pequeno}

Doutorado em Saúde Coletiva - Universidade de Fortaleza - Unifor

E-mail: lupequeno@unifor.br

Paulo Leonardo Ponte Marques

Doutorado em Saúde Coletiva - Universidade de Fortaleza - Unifor

E-mail: paulomarques@unifor.br

\section{RESUMO}

Objetivo: Estimar a prevalência da cárie em crianças na primeira infância sob vulnerabilidade social. Métodos: Pesquisa do tipo transversal realizada no Instituto da Primeira Infância - Iprede, com crianças sob vulnerabilidade social em Fortaleza, Ceará. A coleta de dados foi realizada, em dezembro de 2018 a abril de 2019, com 363 crianças aleatoriamente, por meio de exame bucal e questionário. Os dados foram analisados através de estatística descritiva e do teste qui-quadrado para variáveis categóricas. Resultados: Observou-se uma prevalência de cárie de $35,6 \%$, e que de 1 a 4 lesões representavam a maior porcentagem $(23,7 \%)$. Elevado percentual de crianças nunca foi ao dentista $(81,3 \%)$; semelhante percentual nunca sentiu dor de dente $(82,3 \%)$. Conclusão: Apesar da vulnerabilidade social, a prevalência de cárie foi baixa, e sugere-se realização de ações para minimizar os efeitos da doença na qualidade de vida e desenvolvimento infantil.

Palavras-chave: Cárie Dentária; Criança; Saúde Bucal; Vulnerabilidade Social. 
ABSTRACT

Objective: To estimate the prevalence of caries in children in early childhood under social vulnerability. Methods: Cross-sectional research conducted at the Instituto da Primeira Infância - Iprede, with children under social vulnerability in Fortaleza, Ceará. Data collection was carried out, from December 2018 to April 2019, with 363 children randomly, through oral examination and questionnaire. The data were analyzed using descriptive statistics and the chi-square test for categorical variables. Results: There was a prevalence of caries of $35,6 \%$, with 1 to 4 caries representing the highest percentage $(23,7 \%)$. A high percentage of children never went to the dentist $(81,3 \%)$; a similar percentage never experienced toothache $(82,3 \%)$. Conclusion: Despite the social vulnerability, the prevalence of caries was low, and actions to minimize the effects of the disease on quality of life and child development are suggested.

Keywords: Dental Caries; Child; Oral Health; Social Vulnerability.

\section{INTRODUÇÃO}

A interação entre determinantes de ordem individual e coletiva aponta para diferentes graus de vulnerabilidade, a qual potencializa ou fragiliza o equilíbrio do processo saúde-doença e impacta no desenvolvimento da criança na primeira infância. Dessa vulnerabilidade, emergem múltiplos aspectos negativos que podem repercutir na saúde bucal.1

Os determinantes da estrutura ambiental, social, cultural, econômica e do sistema de saúde são fatores associados no surgimento e evolução da cárie dentária.1 Embora seja inegável o declínio em nível mundial, a cárie dentária ainda é doença crônica comum e que afeta grande parte das crianças na primeira infância, especialmente no período compreendido entre o nascimento até os 6 anos de idade. 2

Devido a sua prevalência e as severas consequências na qualidade de vida é considerada um problema de saúde pública em diversos municípios.3 Estritamente com relação a dentição decídua, verificase um aumento da doença à proporção que aumenta a idade, apesar de dados de prevalência muito variados. 4

No último grande levantamento epidemiológico realizado no Brasil, os resultados indicaram que $27 \%$ das crianças entre 18 a 36 meses possuíam pelo menos um dente com cárie na dentição decídua.5 Dessa forma, essa doença pode ser encarada como um problema de saúde na infância, vista não apenas como um problema dentário específico, mas comprometendo os indivíduos com muitas consequências.6

Com o avançar da idade e consequentemente da doença, o quadro piora. Com 5 anos, as crianças brasileiras apresentam em média 2,43 dentes cariados, ausentes ou restaurados, sendo predominante o componente cariado, responsável por cerca de $80 \%$ do índice. Em regiões de maior vulnerabilidade social, como a Nordeste, esses números são ainda maiores.7

Diante de uma acelerada transição demográfica 8, consequências como a dor de origem dentária afetarão décadas de evolução por influenciarem negativamente na alimentação, fazendo com que as crianças manifestem um lento crescimento, disfunções nutricionais, baixo peso, alterações do sono, além 
de atrapalhar o rendimento escolar e o contato com o meio social, causando uma insuficiência cognitiva e atitudinal.9

Destaca-se a função que a epidemiologia tem como mecanismo fundamental para a vigilância da saúde bucal, uma vez que a realidade brasileira está justamente marcada pela desigualdade na saúde.10 Neste cenário, instituições não governamentais atuam paralelamente para mitigar os riscos que repercutem negativamente no desenvolvimento das crianças na primeira infância, mas nem sempre estas conseguem focar em uma atenção, de forma abrangente, da saúde bucal.

Diante deste cenário, o presente estudo teve como objetivo estimar relações entre a prevalência da cárie dentária em crianças da primeira infância e fatores ligados a situação de vulnerabilidade social.

\section{MÉTODOS}

Trata-se de um estudo observacional, de corte transversal que teve como cenário o Instituto da Primeira Infância - Iprede, situado no município de Fortaleza-Ceará. Implantada na década de 1980, a instituição é classificada como uma organização não governamental voltada para a prevenção e tratamento de crianças sob condição de vulnerabilidade social, com foco no combate à desnutrição e melhoria do desenvolvimento infantil de zero a seis anos de idade.

No segundo semestre de 2019 o instituto possuía mais de 1200 crianças cadastradas, recebendo atendimento em diversas especialidades na área da saúde e das ciências humanas.

A amostra representativa, calculada considerando um erro amostral de 5\% e nível de confiança de 95\%, foi constituída por 363 participantes, os quais foram selecionados aleatoriamente após a aplicação de critérios de inclusão e exclusão. Consideraram-se como critérios de inclusão as crianças da primeira infância (zero a seis anos), cujos responsáveis concordaram em participar da pesquisa, acompanhadas pela instituição. Foram considerados como critérios de exclusão: crianças acima de 6 anos, com condições mentais desfavoráveis.

A coleta de dados foi realizada no período de dezembro de 2018 a abril de 2019 por meio de exame bucal e questionário autorreferido sobre aspectos demográficos e sociais dos participantes, incluindo idade, sexo, raça, escolaridade, renda familiar e estado nutricional emagrecido.

O exame bucal buscou a identificação de lesões de cárie nos dentes presentes na cavidade bucal dos participantes. Foi realizado em um ambiente com iluminação natural na instituição por uma equipe de seis acadêmicos de Odontologia do $9^{\circ}$ semestre de uma universidade privada do Ceará. Os examinadores e anotadores e previamente treinados, calibrados e corretamente paramentados.

Para identificar a presença ou ausência de lesões de cárie foram utilizados espelhos clínicos, gaze, luvas de procedimentos, máscara e abaixadores de língua descartáveis. Os dados foram registrados em uma ficha de coleta individual, utilizada como instrumento de coleta no último Levantamento Epidemiológico 
em Saúde Bucal do Brasil, SB Brasil 2010.7

Os dados foram consolidados, tabulados e organizados no programa SPSS versão $22.0^{\circledR}$, Statistical Package for the Social Sciences (SPSS Inc., Chicago, Estados Unidos). Em seguida foram analisados por meio de estatística descritiva e utilizou-se o teste qui-quadrado, com nível de significância de 5\%, para verificar a associação entre a presença e a ausência de lesão de cárie nas variáveis independentes (sexo, renda da família, estado nutricional emagrecido resultante do balanço entre a ingestão e a perda de nutrientes e o nível de escolaridade dos responsáveis). A prevalência de cárie foi calculada tomando-se a quantidade de participantes com lesão e o total de participantes.

A pesquisa respeitou a Resolução CNS/MS nº 466/2012, e teve aprovação do Comitê de Ética em Pesquisa da Universidade de Fortaleza sob parecer de no 2.790.414, em 30 de julho de 2019.

\section{RESULTADOS}

A amostra consistiu de 185 (51\%) crianças do sexo masculino, $186(51,2 \%)$ na faixa etária de 4 a $\leq$ 6 anos, $212(58,4 \%)$ cor da pele pardo e a maioria das famílias $(200 ; 55 \%)$ participantes detinham renda per capita menor que hum salário mínimo. Cerca de 254 (67,5\%) crianças estão estudando atualmente. Já a porcentagem da escolaridade dos responsáveis era predominante no nível fundamental $(227 ; 62,6 \%)$.

Entre os participantes, a predominância de estado nutricional emagrecido foi alta (198; 54,5\%). Detectou-se forte interação entre mãe e filho $(329 ; 90,6 \%)$ (Tabela 1$)$.

Tabela 1 - Características sociodemográficas relativas às crianças menores ou igual a seis anos e seus dados familiares.

\begin{tabular}{llc}
\hline Variáveis Sociodemográficas & Frequência & $\%$ \\
SEXO & 185 & $51 \%$ \\
Masculino & 178 & $49 \%$ \\
Feminino & & \\
COR/ RAÇA & 212 & $58,4 \%$ \\
Pardo & 86 & $23,6 \%$ \\
Branco & 53 & $14,6 \%$ \\
Preto & 12 & $3,3 \%$ \\
Indígena & & \\
RENDA & 200 & $55 \%$ \\
Menos de 1 salário mínimo & 41 & $11,29 \%$ \\
Igual a 1 salário mínimo & 84 & $23,14 \%$ \\
Mais de 1 salário mínimo & 38 & $10,46 \%$ \\
Não sabe informar & &
\end{tabular}




\section{IDADE}

$\begin{array}{lll}0 \mathrm{a}<2 & 44 & 12,1 \% \\ 2 \mathrm{a}<4 & 133 & 36,6 \% \\ 4 \mathrm{a} \leq 6 & 186 & 51,2 \%\end{array}$

ESTADO NUTRICIONAL EMAGRECIDO

$\begin{array}{lcc}\text { Ausente } & 165 & 45,5 \% \\ \text { Presente } & 198 & 54,5 \% \\ \text { ESTUDANTE } & & \\ \text { Sim } & 245 & 67,5 \% \\ \text { Não } & 118 & 32,5 \%\end{array}$

INTERAÇÃO ENTRE

MÃE E FILHO

$\begin{array}{lll}\text { Presente } & 329 & 90,6 \% \\ \text { Ausente } & 34 & 9,4 \%\end{array}$

ESCOLARIDADE DO RESPONSÁVEL

$\mathbf{O}$

\begin{tabular}{lll} 
Analfabeta & 6 & $1,6 \%$ \\
Fundamental & 227 & $62,5 \%$ \\
Médio & 122 & $33,6 \%$ \\
Superior & 8 & $2,2 \%$ \\
\hline \multicolumn{2}{c}{ Fonte: Dados da pesquisa }
\end{tabular}

Na tabela 2, observa-se alta prevalência de cárie (126; 35,6\%). Existe um grande percentual de crianças que nunca foram ao dentista $(295 ; 81,3 \%)$, igualmente, que nunca sentiram dor no dente (299; $82,3 \%)$.

Com relação a condição de saúde bucal, 243 crianças $(64,4 \%)$ não apresentavam a doença; sendo encontrada no segmento de 1 a 4 lesões de cárie, 86 (23,7\%), embora 4 (1,1\%), tenham mais de 10 lesões (Tabela 2).

Diante da condição da doença cárie, as crianças do sexo masculino $(65 ; 64,9 \%)$ representavam a maioria das que tinham lesão visível.

Quanto a renda familiar, tanto as famílias que tinham melhores condições $(54 ; 65,1 \%)$, quanto as que possuíam uma menor condição financeira $(183 ; 65,4 \%)$ continham um maior número de crianças livres da lesão de tecido cariado. 
Tabela 2 - Análise mostrando as variáveis associadas visitas ao dentista e a gravidade da doença cárie.

\begin{tabular}{lcc} 
Variáveis & Frequência & Porcentagem \\
VISITA AO DENTISTA & 68 & $18,7 \%$ \\
Sim & 295 & $81,3 \%$ \\
Não & & \\
DOR DE DENTE & 64 & $17,6 \%$ \\
Sim & 299 & $82,3 \%$ \\
Não & & \\
LESÕES DE CÁRIE & 243 & $64,4 \%$ \\
Sem lesão & 86 & $23,7 \%$ \\
1 A 4 & 36 & $9,91 \%$ \\
5 A 10 & 2 & $1,10 \%$ \\
Mais de 10 & 4 & $0,27 \%$ \\
Não avaliado & & \\
Não possui dentes & & \\
\hline
\end{tabular}

Fonte: Dados da pesquisa

Na tabela 3 estão apresentadas as variáveis em relação a presença de cárie. Um total de 126 (34,7\%) apresentou lesão de cárie, escolaridade dos responsáveis que tiveram menos de 8 anos de estudo, influenciou, embora não significante estatisticamente $(\mathrm{p}=0,169)$, apresentavam $24,2 \%$ das crianças com lesão de cárie uma diferença de 16,8\% em relação aos que tinha escolaridade acima de 8 anos de estudo.

Tabela 3 - Análise estatística da prevalência de lesão de cárie associado as variáveis socais das famílias participantes.

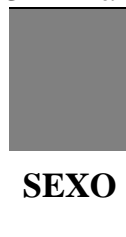

Masculino

Feminino

RENDA

Até 1 salário

Mais de 1 salário

\section{Lesão de \% cárie}

p-valor* cárie 
ESTADO NUTRICIONAL EMAGRECIDO

\begin{tabular}{lccccc} 
Ausente & 55 & $33,3 \%$ & 110 & $66,7 \%$ & \\
Presente & 71 & $35,9 \%$ & 127 & $64,1 \%$ & $\mathbf{0 , 6 1 5}$ \\
$\begin{array}{l}\text { ESCOLARIDADE } \\
\text { RESPONSÁVEIS }\end{array}$ & DOS & & & & \\
\hline$<8$ anos de estudo & 88 & $24,2 \%$ & 145 & $39,9 \%$ & \\
$>8$ anos de estudo & 38 & $10,4 \%$ & 92 & $25,3 \%$ & $\mathbf{0 , 1 6 9}$ \\
\hline
\end{tabular}

Fonte: Dados da pesquisa

Teste qui-quadrado*

\section{DISCUSSÃO}

Diante de um contexto institucional que acolhe crianças em vulnerabilidade social no nordeste brasileiro, os achados apontaram uma maioria de participantes $(64,4 \%)$ livre da doença.

Apesar dos resultados, a cárie dentária continua sendo um problema de saúde pública ${ }^{11}$ e precisa ser levada em consideração para realização de planejamento de sistemas de prevenção que considerem o perfil social, demográfico e epidemiológico.

A cárie é considerada uma das doenças mais disseminada no mundo, apesar de haver uma série de ações preventivas para evitá-la. ${ }^{12}$ Entre essas ações, se encontram aquelas de ampla abrangência como a fluoretação das águas ${ }^{13,14}$, as relacionadas com aspectos modificáveis, como higiene bucal e hábitos alimentares, e aspectos não modificáveis, como socioeconômicos e genéticos pertencentes a essa doença. ${ }^{15}$

A maioria das crianças examinadas que apresentavam a doença possuíam entre 1 e 4 dentes cariados $(\mathrm{n}=86 ; 23,7 \%)$. Os resultados da prevalência de cárie foram inferiores ao encontrado em outro estudo realizado no Brasil, onde cerca de 50\% das crianças na primeira infância apresentaram cárie. ${ }^{16}$ Essa diferença pode estar relacionada às características sociais e demográficas do contexto onde foi realizado o estudo. Além disso, a realização de acompanhamento odontológico pela atenção primária à saúde pode impactar positivamente na redução de lesões cariosas.

Um estudo apontou a relação entre o horário da alimentação das crianças e do horário de dormir, relacionado com o risco à doença cárie. Os autores enfatizam que uma alimentação cariogênica na primeira infância, apresenta o risco de se ter cárie é quase quatro vezes maior, pois alimentações ricas em carboidratos complexos podem ser consideradas o determinante principal da doença nas crianças, fazendo com que acumule nas superfícies dentárias mais açúcares provendo assim um ambiente favorável ao desenvolvimento da doença. ${ }^{17}$

Outro fator é a baixa percepção dos pais, que apesar de reconhecerem suas responsabilidades e 
conhecerem comportamentos corretos em saúde oral, eles não tomam atitudes preventivas para assegurar hábitos de alimentação sem o consumo de alimentos açucarados e de higiene oral diária. ${ }^{11}$

Foi identificada maior prevalência de cárie nos participantes do sexo masculino e que apresentavam de 1 a 4 lesões de cárie na cavidade bucal. Sabe-se que a cárie precisa de vários fatores para evolução e não só da ingestão de alimentos cariogênicos, o que foi visto nesse estudo, já que as crianças tinham alto risco de cárie, má higiene bucal e uma elevada ingestão de alimentos açucarados, por ser uma alimentação mais barata. ${ }^{18}$ Além disso, é comum em famílias em vulnerabilidade social terem uma dieta com predominância de alimentos cariogênicos e sem restrição da quantidade de açúcar ingerido, faz-se necessário o desenvolvimento contínuo de ações individuais e coletivas que busquem minimizar as consequências e impactos negativos ao desenvolvimento infantil. ${ }^{19}$

Os achados mostraram alto índice de crianças que nunca foram ao dentista $(81,3 \%)$ resultando um maior índice de cárie, mesmo que a baixa ingestão de carboidratos diminua consideravelmente o risco de cárie. Estudos apontam que a cárie dentária está entre as principais causas para infecções de origem odontogênica, podendo atingir indivíduos em diferentes faixas etárias e níveis socieconômicos, e ter consequências no desenvolvimento da criança, além de afetar o estado nutricional. ${ }^{16,20}$

Observou-se elevada predominância do estado nutricional emagrecido (54,5\%). Além de danificar a estrutura do dente, a cárie dentária pode agir negativamente no desenvolvimento e crescimento da criança, que ocasiona uma alimentação precária, consequentemente apresentando várias deficiências nutricionais que causam problemas no crescimento. ${ }^{21,22}$ Diante disso, pode ocasionar uma má formação na estrutura dentária, uma maior perda de dentes acarretando problemas oclusais, e outra possibilidade é o comprometimento da mastigação.

Nesse estudo verificou-se que apesar de 18,7\% das crianças já terem ido ao dentista cerca de 17,6\% delas apresentaram dor nos dentes, significando dizer que as visitas ao dentista podem estar relacionadas ao incômodo das crianças. A cárie traz inúmeros danos à saúde das crianças e que vão desde danos físicos aos psicológicos afetando não só a qualidade de vida das crianças, mas também de seus responsáveis. ${ }^{23}$

A observação da doença em crianças da primeira infância é importante não somente pela provável recorrência nos dentes afetados, mas também por distúrbios futuros, como a impactação de dentes permanentes, desenvolvimento de má-oclusões e falta de espaço na arcada dentária. ${ }^{24}$

Entretanto, o tratamento de dentes decíduos é procurado apenas quando a criança tem algum incômodo, já que os responsáveis acreditam que a solução desse problema seja a substituição dos decíduos pelos permanentes. ${ }^{25} \mathrm{E}$ que mesmo apresentando uma alta interação entre mães e filhos, o índice de visitas ao dentista foi baixo, mostrando também que o baixo nível de escolaridade dos pais pode influenciar nesse cuidado. Além disso, a insuficiência de resolutividade de unidades básicas de saúde pode dificultar o acesso dessa população a assistência odontológica. ${ }^{26}$ 
Foi observado que a escolaridade dos responsáveis com menos de 8 anos de estudo se mostrava influenciadora, com maior percentual, embora não tenha sido estatisticamente significante $(\mathrm{p}=0,169)$, na questão da saúde bucal, em que responsáveis com menor escolaridade, tinham filhos com maiores chances de apresentarem cárie. Acredita-se que apesar dos responsáveis entenderem que é necessário seguir as recomendações dos cirurgiões dentistas para o cuidado com a higiene oral das crianças, fatores como nível de compreensão, condição de vida deficiente e dificuldade do acesso aos serviços de saúde, assim como a disponibilidade limitada comprometem o sucesso de medidas de prevenção da doença. ${ }^{16}$

Sabe-se que má nutrição, baixo peso, nível socioeconômico e difícil acesso aos serviços de saúde são fatores de risco para a cárie, além da má higiene bucal. A cárie é uma interação entre fatores determinantes incluindo o dente, a dieta cariogênica, os microrganismos etiológicos e o tempo. Com a higiene bucal precária e o passar do tempo, a cárie vai se desenvolvendo e pode gerar sérios problemas para os indivíduos. ${ }^{11}$ Além desses problemas, pode prejudicar o convívio social das crianças por causa da questão estética, acarretando isolamento e timidez. ${ }^{27}$

Notou-se que a maioria das crianças que apresentaram cárie vivem em um nível socioeconômico baixo, menor que 1 salário mínimo (n=200;55\%), e que o nível de escolaridade dos responsáveis também é baixo o que nos faz entender que o acesso à informação e aos serviços de saúde são mais precários. ${ }^{28} \mathrm{O}$ conhecimento sobre saúde bucal dos responsáveis com essas características socioeconômicas são poucos, mas a repetição da instrução de higiene oral nas consultas, acarretou a iniciação da escovação dental da criança e a mudanças de hábitos. ${ }^{11}$ Nota-se que pais com condições socioeconômicas desfavorecidas, afeta m diretamente na condição de saúde dos filhos, tornando-se um fator de vulnerabilidade à doença.

Diante do exposto, a equipe de saúde bucal pode elaborar mecanismos, como orientar ações de promoção de saúde com as crianças que possuem atividade de cárie e com maior risco a doença, com o intuito de reduzir a prevalência de cárie nas crianças da primeira infância. ${ }^{29,30}$

Foi observado que $81,3 \%$ das crianças analisadas, nunca tinham visitado o dentista, fato que corrobora com o estudo de Roncalli ${ }^{31}$ que, apesar da redução de cárie nas crianças, o número de crianças que nunca trataram essa doença é bem elevado. Este estudo enfatiza que apesar de alguns resultados terem melhorados outros obtiveram uma piora, com por exemplo o índice de dentes obturados e de perdidos respectivamente. ${ }^{31}$ É notório que há uma necessidade de se ter uma atenção maior para obter equidade na Saúde, pois existem regiões onde o índice de cárie é bem maior que em outra. Também foi visto que apesar da diminuição da cárie nos decíduos, $80 \%$ dos dentes cariados não tinham sido tratados.

\section{CONCLUSÃO}

Apesar do contexto institucional com crianças em situação de vulnerabilidade social, o que poderia apontar maior probabilidade da presença de lesões de cárie, foi identificada uma baixa prevalência da 
doença. Sugere-se que sejam realizadas intervenções contínuas assistenciais e de promoção da saúde para minimizar os efeitos da doença na qualidade de vida e desenvolvimento infantil na primeira infância. 


\section{REFERÊNCIAS}

1. PINTO, G.D.S., AZEVEDO, M.S., GOETTEMS, M.L. et al. Are maternal factors predictors for early childhood caries? results from a cohort in southern Brazil. Braz Dent J. 2017 jun; 28(3): 391-397. Disponível em: http://www.scielo.br/scielo.php?script=sci_arttext\&pid=S010364402017000300391\&lng=en. https://doi.org/10.1590/0103-6440201601047.

2. FAUSTINO-SILVA, D.D., COLVARA, B.C., MEYER, E. Et al. Motivational interviewing effects on caries prevention in children differ by income: A randomized cluster trial. Community Dent Oral Epidemiol. 2019 Dec;47(6):477-484. doi: 10.1111/cdoe.12488. Epub 2019 Aug 6. PMID: 31385333.

3. NARVAI, P.C., FRANZÃO, P., RONCALLI, A.G. et al. Cárie dentária no Brasil: declínio, polarização, iniqüidade e exclusão social [Dental caries in Brazil: decline, polarization, inequality and social exclusion]. Rev Panam Salud Publica. 2006 Jun;19(6):385-93. Portuguese. doi: 10.1590/s1020-49892006000600004. PMID: 16968593.

4. HUMBERTO, N.V., BENZAQUEN, P.G. Cárie dentária em crianças de 5 anos: fatores sociodemográficos, lócus de controle e atitudes parentais. Ciênc. saúde coletiva. Available from: http://www.scielo.br/scielo.php?script=sci_arttext\&pid=S1413-

81232017000100191\&lng=en. https://doi.org/10.1590/1413-81232017221.13582015.

5. LIMEIRA, A., LIMA, F.R., FRANCA, C.D. et al. Caries 'prevalence in children and caregivers of a day care center in Recife/PE. Odontol. Clín.-Cient. V.4, n.9, p.325-329, 2010.

6. LEMOS, L.V.F.M., MYAKI, S.I, WALTER, L.R.D.F. et al. Oral health promotion in early childhood: age of joining preventive program and behavioral aspects. Einstein (São Paulo). 2014 jan-mar; 12(1): 6-10. Disponível em: http://www.scielo.br/scielo.php?script=sci_arttext\&pid=S167945082014000100003\&lng=en. https://doi.org/10.1590/S1679-45082014AO2895 .

7. SB Brasil 2010: Pesquisa Nacional de Saúde Bucal: resultados principais / Ministério da Saúde. Secretaria de Atenção à Saúde. Secretaria de Vigilância em Saúde. - Brasília: Ministério da Saúde, 2012.

8. TEIXEIRA, F.O. LAZARETTI, L.R., FILHO, J.M. et al. Fatores associados a produtividade do trabalho e a transição demográfica dos estados brasileiros. Rev de Desenv Econ. V. 3, n. 44, p.172-298, 2019. DOI: $10.36810 /$ rde.v3i44.6236

9. PIOVESAN, C., ANTUNES, J.L., MENDES, F.M. et al. Influence of children's oral health-related quality of life on school performance and school absenteeism. J Public Health Dent. 2012 Spring;72(2):15663. doi: 10.1111/j.1752-7325.2011.00301.x. Epub 2012 Feb 28. PMID: 22372974.

10. BARTOSOVA, M., SVETLAK, M., KUKLETOVA, M. Et al. Emotional stimuli candidates for behavioural interventional in the preservation of early childhood caries: a pilot study. BMC oral health. 2019 fev; 19(1): 33-49.

11. MACAMBIRA, D.S.C., CHAVES, E.S., COSTA, E.C. et al. Conhecimento de pais/cuidadores sobre saúde bucal na infância. Saúde e Pesquisa. 2018 set-dez; 10(3): 463-472. https://doi.org/10.17765/21769206.2017v10n3p463-472

12. BARTOSOVA, M., SVETlAK, M., KUKLETOVA, M. Et al. Emotional stimuli candidates for behavioural intervention in the prevention of early childhood caries: a pilot study. BMC Oral Health. 2019 Feb 18;19(1):33. doi: 10.1186/s12903-019-0718-4. PMID: 30777061; PMCID: PMC6379970. 
13. NARVAI, P.C. Cárie dentária e flúor: uma relação do século XX. Ciênc. saúde coletiva [Internet]. 2000; 5 (2): 381-392. Disponível em: http://www.scielo.br/scielo.php?script=sci_arttext\&pid=S1413-81232000000200011\&lng=en. https://doi.org/10.1590/S1413-81232000000200011 .

14. LACERDA, A.P.A.G., OLIVEIRA, N.A., PINHEIRO, H.H.C. et al. Water fluoridation in the ten largest municipalities of the state of Tocantins, Brazil. Cien Saude Colet. 2020 Mar;25(4):1507-1518. Portuguese, English. doi: 10.1590/1413-81232020254.17722018. Epub 2018 Aug 17. PMID: 32267451.

15. FRIAS, A.C., ANTUNES, J.L.F., JUNQUEIRA, S.R. et al. Determinantes individuais e contextuais da prevalência de cárie dentária não tratada no Brasil. Rev Panam Salud Publica. V.4, n.22, p. 279-85, 2007. 16. CORREA-FARIA, P., PAIXÃO-GONÇALVES, S., PAIVA, S.M. et al. Incidence of dental caries in primary dentition and risk factors: a longitudinal study. Brazilian oral research. 2016 mai; 30(1): 59-68. Disponível em: http://www.scielo.br/scielo.php?script=sci_arttext\&pid=S180683242016000100254\&lng=en. Epub em 20 de maio de 2016. http://dx.doi.org/10.1590/1807-3107BOR2016.vol30.0059.

17. WATANABE, M., WANG, D.H, IJICHI, A. Et al. The influence of lifestyle on the incidence of dental caries among 3-year-old Japanese children. Int J Environ Res Public Health. 2014 Dec 5;11(12):12611-22. doi: 10.3390/ijerph111212611. PMID: 25489996; PMCID: PMC4276635.

18. MONYNIHAN, P.J., KELLY, S.A. Effect on caries of restricting sugars intake: systematic review to inform WHO guidelines. J Dent Res. 2014 Jan;93(1):8-18. doi: 10.1177/0022034513508954. Epub 2013 Dec 9. PMID: 24323509; PMCID: PMC3872848.

19. SILVA, M.das G.B.da.; CATÃO, M.H.C. de V.; ANDRADE, F.J.P. de et al. Early childhood caries: associated risk factors carie precoces de la infancia: factores de resto asociados. Arch Hearth Invest. V. 12, n. 6, p.574,579, 2017. DOI: https://doi.org/10.21270/archi.v6i12.2264

20. DE MELO, Herlla Sofia Sales; DE MELO, Ricardo Eugenio Varela Ayres. Infecções de origem odontogênica: uma revisão de literatura: Infections of odontogenic origin: a review of the literature. Archives of Health, v. 2, n. 4, p. 853-856, 2021.

21. CAMARGO, B., PAVINATO, L.C., CARDOSO, M. Et al. Características de pacientes com cárie severa da infância: análise de pacientes atendidos em centro de referência. Rev Fac de Odontol - UPF. 2018 mai-ago;

23(2):

133-138.

DOI: https://doi.org/10.5335/rfo.v23i2.8776

22. BASIR, L., RASTEH, B., MONTAZERI, A. Et al. Avaliação de quatro níveis da intervenção de promoção da saúde para prevenir a cárie na primeira infância: um ensaio clínico randomizado. BMC Public Health. 2 de outubro de 2017; 17 (1): 767. doi: 10.1186 / s12889-017-4783-9. PMID: 28969655; PMCID: PMC5625785.

23. DO, L.G., SCOTT, J.A., THOMSON, W.MT. et al. Common risk factor approach to address socioeconomic inequality in the oral health of preschool children--a prospective cohort study. BMC Public Health. 2014 May 6; 14:429. doi: 10.1186/1471-2458-14-429. PMID: 24885129; PMCID: PMC4039048.

24. ANTUNES, L.A.A., ORNELLAS, G., FRAGA, R.S. et al. Oral health outcomes: the association of clinical and socio-dental indicators to evaluate dental caries in preschool children. Ciênc \& Saúde 
Coletiva. 2018

fev;

23:

491-500

Disponível

ARCHIVES OF HEALTH

http://www.scielo.br/scielo.php?script=sci_arttext\&pid=S1413-

81232018000200491\&lng=en. https://doi.org/10.1590/1413-81232018232.21022015.

25. VILAR, M.O., PINHEIRO, W.R., ARAUJO, I.deS. Prevalence of dental caries in children in condition of social vulnerability. Id on Line Rev. Mult. Psic. V.14, n. 49 p. 577-587, 2020. DOI: https://doi.org/10.14295/idonline.v14i49.2349.

26. SILVA, R.M.M da., VIERA, C.S., TOSO, B.R.G de O. Et al. Problem-solving capacity in children health care: the perception of parents and caregivers. Acta Paul Enferm. V.4, n.26, 2013. Disponível em: http://www.scielo.br/scielo.php?script=sci_arttext\&pid=S0103-

21002013000400013\&lng=en. https://doi.org/10.1590/S0103-21002013000400013.

27. CANGUSSU, M.C., CABRAL, M.B.B.S., MOTA, E.L.A et al. Fatores de risco para a cárie dental em crianças na primeira infância, Salvador-BA. Rev. Bras. de Saude Matern. Infant. 2016 jan-mar; 16(1): 5765. Available from: http://www.scielo.br/scielo.php?script=sci_arttext\&pid=S 151938292016000100057\&lng=en. https://doi.org/10.1590/1806-93042016000100007.

28. PIVA, F., PEREIRA, J.T., LUZ, P.B. et al. A longitudinal study of early childhood caries and associated factors in Brazilian children. Braz dental j. 2017 apr; 28(2): 241-248. Disponível em: http://www.scielo.br/scielo.php?script=sci_arttext\&pid=S0103-

64402017000200241\&lng=en. https://doi.org/10.1590/0103-6440201701237.

29. LAZZARINM H.C., VACCARI, L., BASSANESI DOS SANTOS, C.C. et al. Avaliação do perfil de prescrição de dentifrícios fluoretados por cirurgiões dentistas em crianças menores de 06 anos. ConScientiae Saude. 2018 mar; 17(1): 32- 40. DOI: https://doi.org/10.5585/ConsSaude.v17n1.7528

30. SCHWENDLER, A., FAUSTINO-SILVA, D.D, ROCHA, C.F. Saúde Bucal na Ação Programática da Criança: indicadores e metas de um Serviço de Atenção Primária à Saúde. Ciênc \& Saúde Coletiv. 2017 jan; 22: 201-207. Available from: http://www.scielo.br/scielo.php?script=sci_arttext\&pid=S141381232017000100201\&lng=en. http://dx.doi.org/10.1590/1413-81232017221.07912015.

31. RONCALLI, A.G. Projeto SB Brasil 2010 - pesquisa nacional de saúde bucal revela importante redução da cárie dentária no país. Cad. Saúde Pública [Internet]. 2011 Jan; 27(1): 4-5. Available from: http://www.scielo.br/scielo.php?script=sci_arttext\&pid=S0102-

311X2011000100001\&lng=en. https://doi.org/10.1590/S0102-311X2011000100001. 